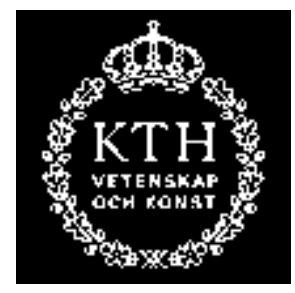

\title{
Theoretical Modeling of Molar Volume and Thermal Expansion
}

\author{
Xiao-Gang Lu
}

Doctoral Dissertation

Department of Materials Science and Engineering Royal Institute of Technology

Stockholm, Sweden 


\begin{abstract}
Akademisk avhandling som med tillstånd av Kungliga Tekniska Högskolan i Stockholm, framlägges för offentlig granskning för avläggande av teknologie doktorsexamen, tisdagen den 7 juni 2005, kl. 10.00 i Salongen, Osquars Backe 31, KTHB, Stockholm.
\end{abstract}

ISBN 91-7178-086-0

ISRN KTH/MSE-05/53-SE+THERM/AVH

(C) Xiao-Gang Lu

Universitetsservice US AB

Stockholm 2005 


\section{Contents}

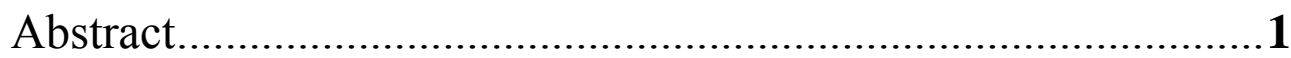

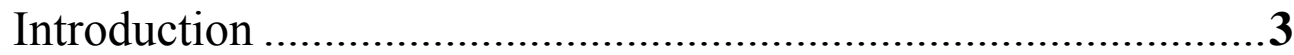

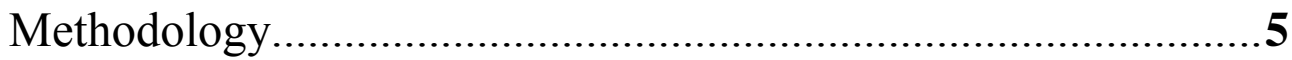

1. First-principles calculations ……………………………………...... 5

2. Debye-Grüneisen model ……………………………………....... 9

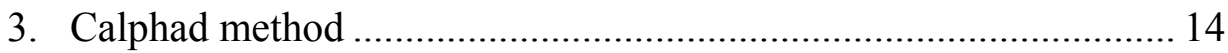

4. Other methods to calculate thermal expansion …………………....... 16

Applications to volume and thermal expansion ..........................19

1. Experimental extrapolations and first-principles calculations.......... 19

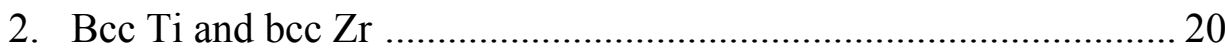

3. Calphad values for unstable structures ………………………........ 21

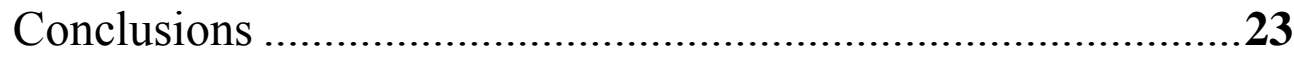

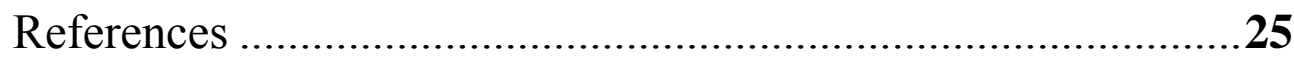

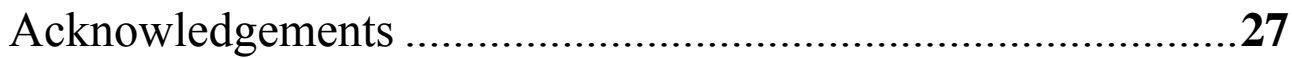

\section{Appended papers}

I. Theoretical modeling of molar volume and thermal expansion X.-G. Lu, M. Selleby and B. Sundman. Acta Mater 53 (2005) 2259.

II. Assessments of molar volume and thermal expansion for selected bcc, fcc and hop metallic elements

X.-G. Lu, M. Selleby and B. Sundman. Calphad, in press, 2005. 
III. Calculations of thermophysical properties of cubic carbides and nitrides using Debye-Grüneisen model

X.-G. Lu, M. Selleby and B. Sundman. Submitted to Acta Materialia.

IV. Implementation of a new model for pressure dependence of condensed phases in Thermo-Calc

X.-G. Lu, M. Selleby and B. Sundman. Calphad, in press, 2005.

\section{Appendix: Other contributions}

Summary of the papers in the Appendix

A. Calculation of the vibrational contribution to the Gibbs energy of formation for $\mathrm{Al}_{3} \mathrm{Sc}$

X.-G. Lu and Y. Wang. Calphad 26 (2002) 555.

B. Thermodynamic assessment of the Ni-Pt system

X.-G. Lu. Licentiate thesis, KTH, Stockholm, 2003.

C. Report on the thermodynamic assessment of the Ni-Al-Pt system X.-G. Lu. HIPERCOAT report, 2005.

D. Reassessment of the T-P phase diagram of iron X.-G. Lu. Licentiate thesis, KTH, Stockholm, 2003. 


\section{Abstract}

Combination of the Calphad method and theoretical calculations provides new possibilities for the study of materials science. This work is a part of the efforts within the CCT project (Centre of Computational Thermodynamics) to combine these methods to facilitate modeling and to extend the thermodynamic databases with critically assessed volume data. In this work, the theoretical calculations refer to first-principles and Debye-Grüneisen calculations.

The first-principles (i.e. ab initio) electronic structure calculations, based on the Density- Functional Theory (DFT), are capable of predicting various physical properties at $0 \mathrm{~K}$, such as formation energy, volume and bulk modulus. The ab initio simulation software, VASP, was used to calculate the binding curves (i.e. equation of state at $0 \mathrm{~K}$ ) of metallic elements, cubic carbides and nitrides. From the binding curves, the equilibrium volumes at $0 \mathrm{~K}$ were calculated for several metastable structures as well as stable structures.

The vibrational contribution to the free energy was calculated using the Debye-Grüneisen model combined with first-principles calculations. Two different approximations for the Grüneisen parameter, $\gamma$, were used in the Debye-Grüneisen model, i.e. Slater's and Dugdale-MacDonald's expressions. The thermal electronic contribution was evaluated from the calculated electronic density of states. The calculated thermal expansivities for metallic elements, cubic carbides and nitrides were compared with Calphad assessments. It was found that the experimental data are within the limits of the calculations using the two approximations for $\gamma$.

By fitting experimental heat capacity and thermal expansivity around Debye temperatures, we obtained optimal Poisson's ratio values and used them to evaluate Young's and Shear moduli. In order to reach a reasonable agreement with the experiments, it is necessary to use the logarithmic averaged mass of the constitutional atoms. The agreements between the calculations and experiments are generally better for bulk modulus and Young's modulus than that for shear modulus.

A new model describing thermodynamic properties at high pressures was implemented in Thermo-Calc. The model is based on an empirical relation between volume and isothermal bulk modulus. Pure Fe and solid $\mathrm{MgO}$ were assessed using this model. Solution phases will be considered in a future work to check the model for compositional dependence. 
Keywords: first-principles calculations, ab initio, Calphad, Debye-Grüneisen model, thermodynamic properties, elastic modulus, volume, thermal expansivity, pressure, Thermo-Calc, VASP, element, carbide, nitride 


\section{Introduction}

Probably volume can hardly be classified as a scientific concept, since its meaning seems so obvious and plain to us, in contrast to the mystical free energy, entropy or phonon. Moreover, it is common sense that an object expands its volume on heating and shrinks on cooling, and thus the term "thermal expansion". However, volume and thermal expansion are indeed profound scientific concepts and deserve extensive and serious studies. In fact, the common sense is not strictly correct as thermal expansivity (i.e. the coefficient of thermal expansion) can be negative due to complex magnetic and phonon behaviors.

Volume and thermal expansivity are basic physical properties when choosing materials for industrial use. It is very often a matter of a balance between performance and density (mass-volume ratio). In aero and automobile industries, more and more materials of low density, such as aluminum, magnesium and titanium alloys, are used to decrease weights, consume less fuel and improve performance. Carbon fiber reinforced materials, with very low density and high strength, are increasingly popular in advanced sport appliances. One example of the applications in industrial production is found in the casting process. The quality control of cast ingots requires good knowledge of densities of melts and solids, as well as their temperature variations.

A family of materials with well-controlled thermal expansion is continuously expanding their application fields, such as biomedical and optical industries, electronic and materials engineering. In substances that have interfaces between different materials, such as composites, coatings and joins, stresses may be generated at the interfaces due to thermal expansion mismatch. Accurate data on the thermal expansion of these materials are required for the selection and development of the systems to improve the adherence to the substrate and for its resistance to thermal stresses. Refractory carbides, nitrides and carbonitrides of transition metals are used as the hard phases in sintered cermet alloys and cemented carbides. It is necessary to know the thermal expansions of the hard phase in relation to the binder phase.

Materials are required to have negligible thermal expansions in an environment with tremendous temperature variations, such as in outer space where the scientific instruments should not be affected by the temperature changes. Careful choice of materials with low thermal expansions is crucial to keep high 
voltage cables from approaching the ground in summer time to avoid electric shock. Invar is one important representative of the materials with negligible thermal expansion. It was discovered one century ago and is still widely used in household appliances and industries, such as cathode-ray tube in TV and computer screens to keep pictures sharp, precise and stable.

Besides various practical applications, measurements of volume and thermal expansion yield useful information for theoretical studies, such as the lattice vibration in crystals, the role of lattice defects in thermal properties, the mechanism of phase transition and anharmonicity which can not be measured directly. For example, the thermal defects are frequently studied by simultaneously measuring the expansion of the lattice parameters and macroscopic length changes of the samples, the formation energy and entropy of the defects can be calculated from the differences between the two types of expansions. Volume and thermal expansivity are also essential parameters of equation of state. Accurate data can facilitate the studies of other parameters in the equations.

A comprehensive and accurate database on volume and thermal expansivity is being developed within the present project. As it is combined with other thermodynamic databases by sharing the same modeling formalism (e.g. the compound energy formalism), its area of application is greatly broadened. In developing a complete thermodynamic database, one major problem arises from numerous metastable and unstable compounds or alloy phases, for which experimental data are unavailable in many cases. Even for stable phases, experimental data are sometimes scattered and, in the worst case, contradictory. One of our goals is to use theoretical methods to predict thermodynamic properties of metastable phases and stable phases with experimental difficulties. 


\section{Methodology}

In the present work, the theoretical methods used are first-principles calculations and the Debye-Grüneisen model. First-principles calculations require no experimental information as an input and are free of fitting parameters, while most of the parameters in the Debye-Grüneisen model can be evaluated from firstprinciples calculations. The Calphad approach is classified as a semi-empirical method using both theoretical models and experimental data.

Atomic spacing (or lattice parameter) is closely related to atomic forces or potential energy, as well as thermodynamic properties such as free energy, entropy and enthalpy. In the theoretical methods, the calculations of volume and thermal expansion are always associated with other physical properties. In contrast, volume can be separately assessed using the Calphad approach and it is possible to develop a stand-alone database. However, the application of such a stand-alone database is limited. In a complete thermodynamic database including volumes, the free energy due to external pressure and temperature-pressure phase diagram can be calculated. Therefore in this section, a brief review will be pursued in a general manner, rather than focusing on volume and thermal expansion.

\section{First-principles calculations}

Although the majority of the methods or theories described below are not used in the present work, knowledge of the first-principles theories and their explanations to the natural phenomena is essential to gain an insight into volume and thermal expansion, and to facilitate the Calphad assessments and the DebyeGrüneisen calculations. This section begins with zero-temperature calculations for stoichiometric, non-stoichiometric compounds and random alloys. Then the lattice vibration at finite temperatures is discussed concerning harmonic approximation and anharmonic effects.

Most modern first-principles calculations are based on the celebrated Density-Functional Theory (DFT) (Hohenberg and Kohn, 1964; Kohn and Sham, 1965). This theory maps a realistic multi-particle system to a one-particle system subjected to an effective external potential. The complex quantum-mechanical problems are thus simplified to solving Schrödinger-like equations for noninteracting single particles. The actual calculations are performed in a self- 
consistent way, i.e., starting from a guessed electronic density, by solving the eigenvalue problem a new electronic density is calculated, and then this procedure is repeated until a converged electronic density is obtained.

First-principles calculations at zero-temperature are nowadays routinely utilized to explain various physical phenomena, and to provide values for various physical properties quantitatively. The accuracy and efficiency of the calculations are improving steadily. In principle, these calculations are generally applicable to any structure and any element in the periodic table. Thus, the calculations of metastable or unstable phases are expected to be reliable.

In the widely used compound energy formalism (CEF), the properties of the end-member compound are of primary importance, since these properties concern the interactions between nearest-neighbor atoms. Proper values even for metastable or unstable end-members must be given in using the models. The common practice is to use as an approximation the formation energy or volume from first-principles calculations when lacking in experimental data. Fortunately, the end-member compounds are perfectly ordered structures (although sometimes metastable or unstable), which belong to the simplest category that first-principles calculations can handle. Some systematic calculations on elements and compounds are given in the following paragraph.

In an early work by Skriver (1985), systematic calculations for pure elements have been performed to examine the total-energy variations along the periodic table. The subsequent work (Jóhannesson, 2002) results in a public database, which has been put on the website at the Center for Atomic-scale Materials Physics (CAMP) at the Technical University of Denmark (DTU). This database contains physical properties of solid metallic elements and compounds obtained by first-principles electronic structure calculations. Recently, Wang et al. (2004b) recalculated the total energies of 78 pure elements and compared with the lattice stability from SGTE (the Scientific Group Thermodata Europe) database. Ghosh and Asta (2005) calculated equilibrium cohesive properties of 69 intermetallic compounds in the $\mathrm{Al}-\mathrm{TM}(\mathrm{TM}=\mathrm{Ti}, \mathrm{Zr}$ and $\mathrm{Hf}$ ) systems. The calculated zero-temperature formation energies generally agree well calorimetric data within a few $\mathrm{kJ}$ per mol, and the calculated lattice parameters agree with experimental data at ambient temperature within $1 \%$.

In Fig. 1, the calculated molar volumes of selected transition metals are shown. Since the thermal expansion below room temperature is low, it is common and reasonable to compare the zero-temperature values with the experimental data at $298 \mathrm{~K}$. 


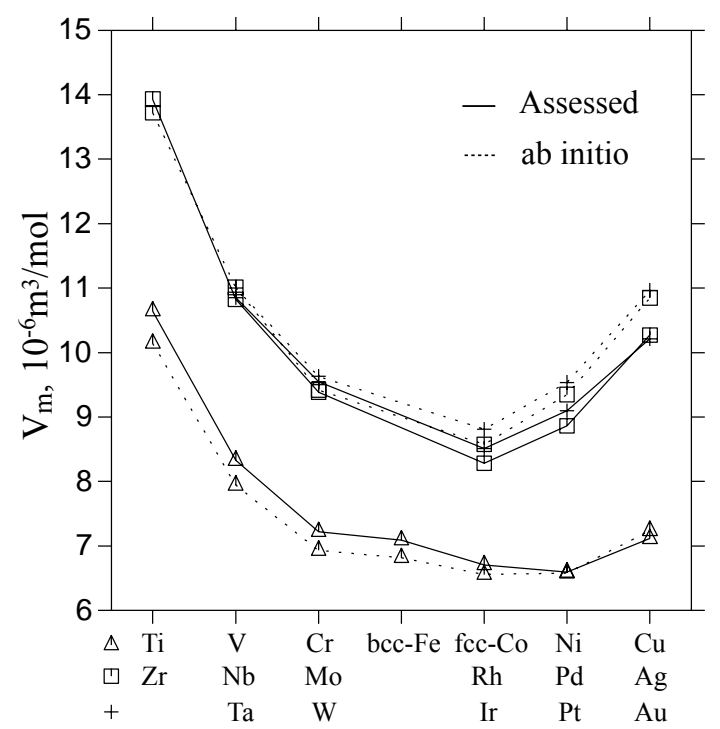

Fig. 1 Molar volumes of transition cubic metals calculated by ab initio calculations (dotted lines) and the Calphad assessments (solid lines).

However, in alloy systems, the properties of disordered solution phases and non-stoichiometric compounds are equally important. First-principles methods have been developing to treat random alloys and compounds with defects. The straightforward but computationally intensive method is the calculations using supercells to simulate random alloys. There are more efficient methods, such as Connolly-Williams method (Connolly and Williams, 1983), special quasirandom structure (SQS) method (Zunger et al., 1990), and coherent-potential approximation (CPA) (Soven, 1967; for the latest development on EMTO-CPA, see Vitos et al., 2001; a latest work on the elastic properties of $\mathrm{Al}_{1-\mathrm{x}} \mathrm{Li}_{\mathrm{x}}$ substitutional random alloys, see Taga et al., 2005). These methods are also used to deal with constitutional point defects. One of the frequently studied compounds is B2 NiAl which contains high concentrations of constitutional point defects and shows large deviations from stoichiometric composition. Korzhavyi et al. (2000) calculated the formation energies of point defects in NiAl using an order N, locally self-consistent Green's function method. The calculated formation energies and lattice parameters agree reasonably with the measurements on the non- 
stoichiometric NiAl. Jiang et al. (2005) used SQS calculations to better reproduce the experimental data in a wider composition range of $\mathrm{B} 2 \mathrm{NiAl}$.

To calculate phase stability, phase equilibrium, as well as thermodynamic properties at finite temperatures, the central issue is to calculate free energy including various contributions coming from configuration, lattice vibration and electronic excitations etc, among which the lattice vibration is the most important and most difficult one to handle from a first-principles point of view.

For harmonic systems, the calculation of free energy is conceptually feasible, although practically computational efforts may be great. One of the successful approaches is linear-response method based on the density-functional perturbation theory (Baroni et al., 1987; Baroni et al., 2001), by which the phonon frequencies can be calculated. It is then straightforward to calculate Helmholtz energy. However, the harmonic approximation encounters a fatal problem when modeling thermal expansion. In other words, a harmonic system does not have thermal expansion and anharmonic effects must be considered. A simple and easy amendment to the harmonic approximation is the quasiharmonic approximation, which allows the phonon frequencies to be dependent on volume. Recent works have successfully applied such calculations on Al, Li and Na (Quong and Liu, 1992), Ag (Xie et al., 1999), Al and W (Debernardi et al., 2001), and Ni (Xie et al., 2000; Wang et al., 2004c), as well as stoichiometric compounds $\mathrm{NiAl}, \mathrm{Ni}_{3} \mathrm{Al}$ (Wang et al., 2004c; Arroyave et al., 2005). These calculations show that the quasiharmonic approximation is applicable up to the melting points, except for $\mathrm{W}$ in the calculation by Debernardi et al. (2001), who attributed the underestimated thermal expansivity of $\mathrm{W}$ above $1500 \mathrm{~K}$ to the failure of the quasiharmonic approximation.

It is worth mentioning (though slightly distracts from the main subject here) that the Debye-Grüneisen model used in the present work also adopts the quasiharmonic approximation. Since the Debye temperature is actually a measurement of average phonon frequencies, in the Debye-Grüneisen model, it is the Debye temperature that varies with volume through the Grüneisen parameter. Our calculations for the cubic transition metals and for cubic carbides and nitrides validate the quasiharmonic approximation up to the melting points with a few exceptions for bcc Cr, Mo and W.

A more elaborate way to consider the anharmonic effect is the so-called thermodynamic integration scheme using first-principles molecular-dynamics simulations. This method is applicable to account for the anharmonicity in solids, and is used to calculate the free energy of liquids. One example is the first- 
principles calculations of the melting of Al (de Wijs et al., 1998). The calculated melting temperature and the volume of fusion were $890 \pm 20 \mathrm{~K}$ and $1.26 \pm 0.2$ $\AA^{3} /$ atom, respectively, in comparison with the experimental data of $933.47 \mathrm{~K}$ and $1.24 \AA^{3} /$ atom. They concluded that this calculation strategy is expected to be applicable to a wide range of liquid metals. Similar work has been done to calculate the melting curve of iron at the Earth's core (Alfè et al., 1999), and chemical potentials of solid and liquid solutions of the Earth's core (Alfè et al, 2002).

A series of calculations have been performed to study the vibrational entropy in alloy systems, showing that the effect of lattice vibrations on phase stability can be significant (van de Walle and Ceder, 2002, and references therein).

However, the first-principles treatments of lattice vibrations are computationally time-consuming and, hence, not efficient for a systematic study of a large number of metals and compounds in a database development. At the same time, the accuracy of these calculations may not be able to meet the demands of engineering and industrial applications.

\section{Debye-Grüneisen model}

The Debye model assumes that the solid is an elastic continuum in which the sound velocity is independent of the wavelengths of the traveling sound waves. It uses the acoustic phonon branches to represent all the vibrational spectra. The original Debye model is a harmonic model by treating the vibrating lattice as a set of harmonic oscillators vibrating near their equilibrium positions. As mentioned above, in order to model thermal expansion, anharmonic effects must be considered.

Moruzzi et al. (1988) used quasiharmonic approximation to include the volume dependence of the Debye temperature and thus accounted for the anharmonic effects. They successfully applied the model to evaluate coefficients of linear thermal expansion (CLE) of 14 nonmagnetic cubic metals below $300 \mathrm{~K}$. Moruzzi's method was adopted in many studies hereafter (Sanchez et al., 1991; Herper et al., 1999; Ostanin and Trubitsin, 1998).

The Debye temperature and volume relation used by Moruzzi et al. is:

$$
\theta_{D} / \theta_{D, 0}=\left(V_{0} / V\right)^{\gamma}
$$


where $\theta_{D, 0}$ is the Debye temperature corresponding to the equilibrium volume $V_{0}$ at $0 \mathrm{~K}$. The Grüneisen parameter, $\gamma$, as well as $\theta_{D, 0}$ and $V_{0}$, can be derived from the calculated total-energy binding curve, i.e. $E(V)$, at $\mathrm{T}=0 \mathrm{~K}$.

However, Eq. (1) implies that

$$
\gamma=-\frac{\ln \theta_{D}-\ln \theta_{D, 0}}{\ln V-\ln V_{0}},
$$

which is only an approximation of the definition of the Grüneisen parameter,

$$
\gamma(V)=-\frac{\partial \ln \theta_{D}}{\partial \ln V}
$$

This approximation is valid in the vicinity of $V_{0}(\mathrm{~T}=0 \mathrm{~K})$. Moruzzi et al. showed that the theoretical calculated CLEs agree well with experiments below $300 \mathrm{~K}$. However, at larger volumes (correspond to higher temperatures), large discrepancies may arise. Recently, Wang et al. (2004a) realized this problem and derived a general expression for the relation between $\theta_{D}$ and volume from a meanfield potential. In the present work, we show that it is possible to derive an equivalent $\theta_{D^{-}} V$ relation from the above definition for $\gamma$ in a straightforward way, and also to incorporate it into the Debye-Grüneisen model instead of Eq.(1).

Three well-known approximations used to account for the Grüneisen parameter $\gamma$ can be combined into the following expression (Zharkov and Kalinin, 1971; Wasserman et al., 1996),

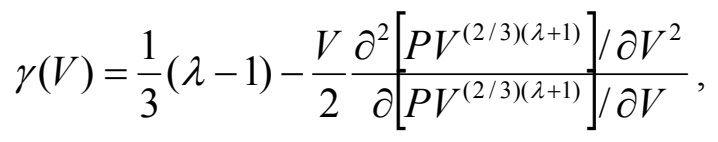

where $P(V)=-\frac{\partial E(V)}{\partial V}$. When $\lambda=-1,0$ and +1 , one obtains the Slater approximation (Slater, 1939), Dugdale-MacDonald approximation (Dugdale and MacDonald, 1953) and the expression resulting from the free volume theory (Vashchenko and Zubarev, 1963), respectively.

When integrating $\gamma(V)=-\frac{\partial \ln \theta_{D}}{\partial \ln V}$ from both sides and using Eq.(4), one obtains 


$$
\theta_{D}(V)=D V^{2 / 3}\left[-\frac{\partial P(V)}{\partial V}-\frac{2(\lambda+1)}{3} \frac{P(V)}{V}\right]^{1 / 2},
$$

where $D$ is the integral constant that is determined as described below.

The Debye temperature is proportional to the sound velocity, $v_{\mathrm{D}}$ (see, e.g. Grimvall, 1999):

$$
\theta_{D}(V)=\frac{\hbar}{k_{B}}\left(\frac{6 \pi^{2} N}{V}\right)^{1 / 3} v_{D}=\frac{\hbar}{k_{B}}\left(\frac{6 \pi^{2} r N_{A}}{V}\right)^{1 / 3} v_{D},
$$

where $v_{D}=k(v) \sqrt{\frac{B}{\rho}}=k(v) \sqrt{\frac{V B}{r m}}$,

and $k(v)=\left\{\frac{2}{3}\left[\frac{2(1+v)}{3(1-2 v)}\right]^{3 / 2}+\frac{1}{3}\left[\frac{1+v}{3(1-v)}\right]^{3 / 2}\right\}^{-1 / 3}$,

where $v$ is Poisson's ratio, $\rho$ is the density, $\mathrm{r}$ is the number of atoms in the chemical formula of the material ( $\mathrm{r}=1$ for an element, 2 for cubic carbides and nitrides), $B$ the bulk modulus. The mass, $\mathrm{m}$, is an effective atomic mass defined as the logarithmic average of all masses in the formula (Grimvall, 1999). For cubic carbides and nitrides $\mathrm{A}_{1} \mathrm{~B}_{1}, \mathrm{~m}$ is calculated by $\ln (m)=\frac{1}{2} \ln \left(m_{A}\right)+\frac{1}{2} \ln \left(m_{B}\right)$. The choice of the logarithmic average of mass, instead of the arithmetic average, is essential to account for the Poisson's ratio for materials with large differences in atomic masses, such as $\mathrm{TaC}$ and HfC. Fig.2 demonstrates the effect of the average masses on the calculated heat capacity of TaC. For CLE, the influence is very similar. 


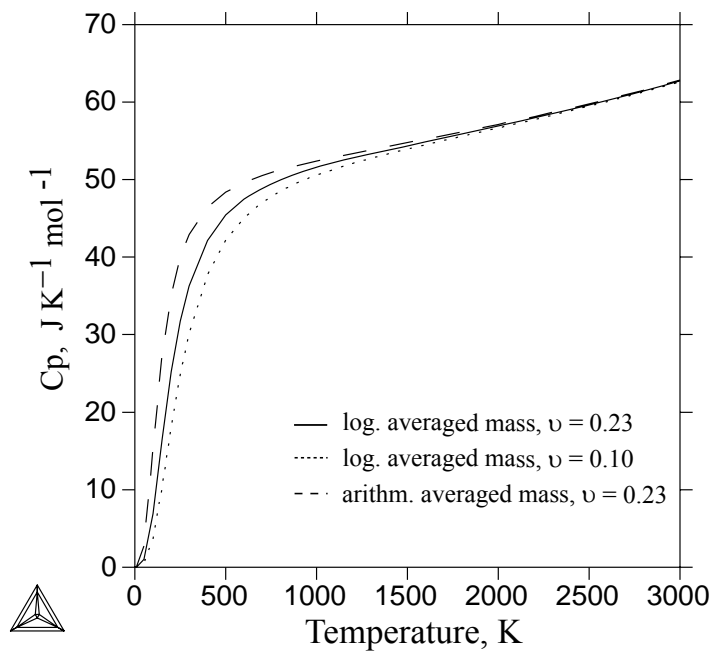

Fig. 2 The influences of mass and Poisson's ratio on the heat capacity of TaC. The influences on CLE are similar.

Using the definition of $B$, one can derive:

$$
\begin{aligned}
& \theta_{D}(V)=k(v) \frac{\hbar}{k_{B}}\left(\frac{6 \pi^{2} N}{V}\right)^{1 / 3}\left[\frac{-V^{2} \frac{\partial P(V)}{\partial V}}{r m}\right]^{1 / 2} \\
& =k(v) \frac{\hbar}{k_{B} \sqrt{r m}}\left(6 \pi^{2} N\right)^{1 / 3} V^{2 / 3}\left[-\frac{\partial P(V)}{\partial V}\right]^{1 / 2}
\end{aligned}
$$

On the other hand, when $\lambda=-1$, i.e. Slater's expression is chosen, Eq.(5) reduces to:

$$
\theta_{D}(V)=D V^{2 / 3}\left[-\frac{\partial P(V)}{\partial V}\right]^{1 / 2}
$$

Comparing Eqs. (9) and (10), the constant $D$ is identified as:

$$
D=k(v) \frac{\hbar}{k_{B} \sqrt{r m}}\left(6 \pi^{2} N\right)^{1 / 3}
$$


Inserting it back into Eq.(5), we obtain

$$
\theta_{D}(V)=k(v) \frac{\hbar}{k_{B} \sqrt{r m}}\left(6 \pi^{2} N\right)^{1 / 3} V^{2 / 3}\left[-\frac{\partial P(V)}{\partial V}-\frac{2(\lambda+1)}{3} \frac{P(V)}{V}\right]^{1 / 2}
$$

This is the general $\theta_{D^{-}} V$ relation we finally reach. Using this relation in the Debye formula as follows, one can express the vibrational free energy as a function of volume and temperature,

$$
\begin{aligned}
& E_{D}(T, V)=\frac{9}{8} N k_{B} \theta_{D}+3 N k_{B} T D\left(\frac{\theta_{D}}{T}\right), \\
& S_{D}(T, V)=3 N k_{B}\left[\frac{4}{3} D\left(\frac{\theta_{D}}{T}\right)-\ln \left(1-e^{-\theta_{D} / T}\right)\right],
\end{aligned}
$$

where $D\left(\theta_{D} / T\right)$ denotes the Debye function. When the free energy is expressed as function of $T$ and $V$, the equilibrium $V(T)$ and thus CLE can be calculated through the minimum of $F(T, V)$, i.e. $\partial F(T, V) / \partial V=0$. Additionally, other thermodynamic properties, such as heat capacity and entropy, can be calculated once the equilibrium volume is determined.

By fitting experimental heat capacity and thermal expansivity around the Debye temperature, it is possible to obtain optimal Poisson's ratio values. Using the calculated bulk modulus and Poisson's ratio, one can evaluate Young's and Shear moduli. The agreements between the calculations and experiments for bulk modulus and Young's modulus are generally better than those for shear modulus. The appended Paper III presents a detailed discussion.

The success of the Debye-Grüneisen model rests on two factors. First, reasonable approximations for the Grüneisen parameter are necessary. For cubic elements, carbides and nitrides, the calculations using the Slater and DugdaleMacDonald's approximation set upper and lower limit for CLE and heat capacity. Further work is planned to verify if this conclusion holds for other structures and other materials. Second, only a certain average of the phonon frequencies is needed to calculate certain thermophysical properties (Grimvall, 1999). So even the Debye-Grüneisen model greatly simplifies the phonon spectra, it is still able to catch the essential features of the lattice vibrations. 
In a review by van de Walle and Ceder (2002), they discussed the effect of lattice vibrations on substitutional alloy thermodynamics, and summarized the origin of the vibrational entropy differences between phases with different degrees of ordering, i.e. the "bond proportion" effect, the volume effect and the size mismatch effect. For the volume effect, they stated that "Disordering is typically associated with an increase in volume. Since chemical bonds tend to soften as they lengthen, an overall increase in volume should be associated with a corresponding decrease in the frequency of all phonon modes, resulting in an increase in vibrational entropy". The Debye model and its derivatives, for instance, the DebyeGrüneisen model, can describe the vibrational entropy differences due to this volume mechanism. But for entropy differences due to the changes of the highfrequency phonon spectra, the Debye-like models may fail and even lead to an incorrect prediction of the vibrational entropy. The same problems can arise when calculating volume and thermal expansion, further work is needed to investigate the effects. But how to deal with the high-frequency part of the phonon spectra is indeed a problem for Debye-like models.

\section{Calphad method}

It is still a long way ahead to reach the goal that materials can be designed directly from first-principles calculations without any experimental inputs. In the mean time, the Calphad technique is a perfect complement. By formulating the thermodynamic properties based on thermodynamic rules and by choosing proper physical model, all sorts of experimental data may be used simultaneously in the Calphad method to obtain a consistent description of thermodynamic properties.

The model used to describe the molar volume at ambient pressure can be derived from the definition of the coefficient of linear thermal expansion (CLE) $\alpha$ as:

$$
V_{m}(T)=V_{0} \exp \left(\int_{T_{0}}^{T} 3 \alpha d T\right)+\Delta V_{m}^{m a g n}(T)
$$

where $\mathrm{V}_{0}$ is the molar volume at the reference temperature, $\mathrm{T}_{0} . \Delta \mathrm{V}_{\mathrm{m}}{ }^{\text {magn }}$ is the magnetic contribution to the molar volume. For cubic elements, $3 \alpha$ is the coefficient of volumetric thermal expansion of a phase in the nonmagnetic state. 
Traditionally the Calphad method has been used to describe thermodynamic properties well above room temperature. In the present work, a simple polynomial is used to model nonmagnetic volumetric expansivity above 298 $\mathrm{K}$ :

$$
3 \alpha=a+b T+c T^{2}+d T^{3}+e T^{-2},
$$

where the coefficients are evaluated from experimental data.

The magnetic contribution to the molar volume was treated by Guillermet (1987) by taking the pressure dependence of the Curie temperature, $T_{c}$, into account, i.e.

$$
T_{c}(P)=T_{c}(P=0)+\omega P
$$

where $\omega$ is a constant, and

$$
\Delta V_{m}^{m a g n}(T)=\Delta H_{m}^{m a g n} \frac{d \ln \left(T_{c}\right)}{d P}
$$

where $\Delta H_{m}^{m a g n}$ is the magnetic enthalpy which is described by the model proposed by Inden (1976) and later simplified by Hillert and Jarl (1978). In Hillert and Jarl's model, the Gibbs energy is formulated as a function of $T_{c}$ and the magnetic moment $\beta$, and $\Delta H_{m}^{m a g n}$ can be calculated accordingly.

The pressure effects of volume and CLE are described by various equation of state. In the present work, a model based on the empirical relation suggested by Grover et al. (1973) was derived representing the Gibbs energy due to the pressure effect. For the detailed derivation, the readers are referred to the appended Paper IV in this thesis.

In a Calphad assessment, the choice of experimental data is critical. Generally, the experimental data with high precisions are given high weights, and the program will fit these data with high priority. Experimental data which have low precision will be given low weights and may not be fit equally well. In practice, setting reasonable weights to experimental data is a personal judgment. When two sets of data are both fitted satisfactorily, they are regarded as consistent. Using the Calphad approach inconsistent experimental data are easily identified. 
Inconsistent data should be treated carefully, and new experiments can be performed to examine the questionable data.

In Calphad assessments, extrapolation methods are frequently used to study metastable phases. For example, the properties of metastable phases can be estimated by systematically studying the phases with the same structure in different systems and making extrapolations. One can also extrapolate the properties of metastable phases from their stable regions in the composition-temperaturepressure space, i.e. from related higher-order systems (composition space), or from high temperature or pressure region. For instance, hcp Fe is not stable at ambient pressure; its properties can be estimated from the hcp phase in binary systems containing the element Fe, such as Fe-Ru system. It is also common to extrapolate volume, CLE, bulk modulus and so on, from high pressure region where hep $\mathrm{Fe}$ is stable.

The predictability of the Calphad method is limited by the reliability of the experimental data, and by the fact that the model parameters are usually polynomial even though the model itself is physical. Simple extrapolation may thus not be reliable outside the experimental range, and should be treated carefully by comparing with the values from other methods.

\section{Other methods to calculate thermal expansion}

One can calculate the Debye temperature from the elastic stiffness constants $\mathrm{c}_{\mathrm{ij}}$, which can be derived from the total energies for different distortions of the crystal structure in the first-principles calculations. These calculated elastic constants are corresponding to single-crystal elastic properties. To estimate elastic moduli for polycrystalline materials, the Voigt-Reuss-Hill (VRH) approximation is widely used (Grimvall, 1999). The elastic moduli are in turn used to calculate the transverse and longitudinal sound velocities, from which the average sound velocity can be accurately evaluated for isotropic materials as shown by Anderson (1965). The Debye temperature is calculated using the average sound velocity, instead of using bulk modulus in the present work. The Grüneisen parameter, $\gamma$, can be evaluated using similar methods as in the present work. The CLE is obtained using the following relation,

$$
\alpha=\frac{\gamma C_{V}}{3 B V}
$$


where $\mathrm{C}_{\mathrm{V}}$ is the heat capacity at constant volume, and $\mathrm{B}$ and $\mathrm{V}$ are bulk modulus and volume, respectively.

Wolf and Podloucky (1999) and Mayer et al. (2003) calculated elastic constants and used this method to calculate CLEs of refractory carbides and nitrides, and Laves phases, respectively.

There are several semiempirical models which contain fitting parameters determined by experimental data. Among them, a modified Einstein model developed by Reeber (1975) uses several Einstein frequencies to simulate the phonon spectra of studied materials. The Einstein temperatures and extra fitting constants are fitted to the experimental data. This method has been used to study the thermal expansivities of $\mathrm{NaCl}$ (Wang and Reeber, 1996a), $\mathrm{Cu}$ (Wang and Reeber, 1996b), V, Nb, Ta, Mo and W (Wang and Reeber, 1998). 
- 18 - 


\section{Applications to volume and thermal expansion}

Both the Calphad method and first-principles calculations have made significant progress during the last three decades. Successful applications have been described in the above sections. However, both methods have their limitations and can benefit from their counterparts. On one hand, by using proper thermodynamic models and analyzing various experimental data, the Calphad method obtains phenomenological descriptions of multi-component systems, thus enables thermodynamic and kinetic studies in complex systems and promotes the industrial applications. The efficiency and accuracy on the engineering level is what modern first-principles calculations can not yet reach. On the other hand, first-principles calculations analyze energies and forces at the atomic level, and provide physical knowledge on thermodynamic modeling, especially the information in metastable regions where the Calphad assessment may not be reliable. Very often, it is the physical picture the first-principles calculations revealed that is most important.

In the present work on a volume database, the Calphad method is combined with first-principles calculations and the Debye-Grüneisen model. A few case studies are presented below.

\section{Experimental extrapolations and first-principles calculations}

Prinz (1985) synthesized the metastable bcc Co by use of molecular-beam epitaxial growth to stabilize the crystal structure. Although bcc is the metastable structure for pure $\mathrm{Co}$, it is stable over a wide composition range in the $\mathrm{Fe}-\mathrm{Co}$ system. From the extrapolation of the lattice parameters of the stable bcc Fe-Co alloys, Prinz obtained a 'predicted' value to confirm the experimental result. The values as well as the calculated one from first-principles are listed in Table 1. The differences are merely $1 \%$, which is the typical error for the first-principles calculations for lattice parameter. 
Table 1. Lattice parameter of the metastable bcc Co.

\begin{tabular}{lc}
\hline Methods & Lattice parameter, $\AA$ \\
\hline VASP (this work) & 2.808 \\
Experiment (Prinz, 1985) & 2.827 \\
$\begin{array}{l}\text { Extrapolation from Fe-Co } \\
\text { alloys (Prinz, 1985) }\end{array}$ & 2.819 \\
\hline
\end{tabular}

However, a good agreement is not always reached between the experimental extrapolations and the first-principles calculations. For example, hcp $\mathrm{Fe}$ is not stable at ambient pressure and its volume is extrapolated from highpressure measurements. The molar volume of hcp Fe extrapolated by Anderson et al. was $6.73 \mathrm{~cm}^{3} / \mathrm{mol}$ (Anderson et al., 2001), while the first-principles calculations give a very low value, for instance, $6.12 \mathrm{~cm}^{3} / \mathrm{mol}$ (Wang et al., 2004b, pseudopotential), and $6.19 \mathrm{~cm}^{3} / \mathrm{mol}$ (Bose et al., 2003, FP-LMTO). Magnetism may be a key to eliminate this unusually large discrepancy (near $10 \%$ for volume, corresponding to $3.2 \%$ for lattice parameter) and the above calculated values are for nonmagnetic Fe.

\section{Bcc Ti and bec Zr}

The bcc $\mathrm{Ti}$ and bcc $\mathrm{Zr}$ are not stable below $1155 \mathrm{~K}$ and $1139 \mathrm{~K}$, respectively at atmospheric pressure. Thus the measured lattice parameters around room temperature correspond to the hcp structure. However, by quenching hightemperature bcc alloys to room temperature, it is possible to obtain retained metastable bcc phases. Grad et al. (1995) performed neutron scattering study of the quenched $\mathrm{Zr}-\mathrm{Nb}$ bcc alloys and found a linear relation between the lattice parameter and the alloy composition at room temperature. Combining with previous data, they extrapolated to pure bcc $\mathrm{Zr}$ and obtained a value of the lattice parameter at room temperature. Aurelio et al. (2002) used the same method to estimate the lattice parameter of metastable bcc $\mathrm{Ti}$ at room temperature. In another work, Aurelio et al. (2003) determined the lattice parameters of bec $\mathrm{Zr}$ at several 
temperatures below $900 \mathrm{~K}$. The assessments of CLEs for bcc Ti and bcc $\mathrm{Zr}$ are based on the extrapolated lattice parameters as well as the measurements in their stable regions.

As discussed in the appended Paper I and III, the Debye-Grüneisen calculations using Slater's and Dugdale-MacDonald's expressions show good agreements for most of the metals, carbides and nitrides studied. However, for bcc $\mathrm{Ti}$ and bcc Zr, this is not the case. The assessed CLEs are much lower than the Debye-Grüneisen calculations with Slater's and Dugdale-MacDonald's expressions. One conjecture is that bcc $\mathrm{Ti}$ and $\mathrm{Zr}$ are mechanically unstable at zero temperature, and the phonon anomalies may cause the unusual behaviour.

\section{Calphad values for unstable structures}

Wang et al. (2004b) recalculated the total energies of 78 pure elements and compared with the lattice stability from SGTE database based on Calphad assessments. They found significant discrepancies for most of the transition elements, which were attributed to mechanical instability with respect to Bain/tetragonal distortion. Since the unstable structures can be stabilized by alloying with other elements at certain composition ranges in binary systems, the lattice stabilities of unstable structures can be obtained by extrapolations from the energies of the stable or metastable binary alloys based on either first-principles calculations or experiments.

The same discrepancies may arise for volume and CLE. Since a minor vibration will destroy the unstable lattice, the physical values of volume and CLE are not well defined. However, the mathematical values needed for Calphad modeling can be extrapolated from the volume and CLE of binary alloys in their stable or metastable regions. If the experimental data are not available, firstprinciples calculations or the Debye-Grüneisen model can be used to estimate reasonable values. This method will be applied in the ongoing molar-volume database development. 
$-22-$ 


\section{Conclusions}

Currently, the Calphad technique is the most powerful approach to study thermodynamic and kinetic problems in complex multicomponent systems. However, it relies heavily on experiments and its power can be enhanced enormously if coupled with first-principles calculations. The physical picture depicted by first-principles calculations can facilitate the choice of models and the development of new models within the Calphad approach. The theoretically calculated results may directly be used to pin down key model parameters in Calphad assessments thus reducing the amount of guesswork and dismissing unreasonable assumptions. Alternatively, the calculated results may be treated as "experimental data", crucial for metastable and unstable phases. 
-24 - 


\section{References}

Alfè D, Gillan MJ, Price GD. Nature 401 (1999) 462.

Alfè D, Gillan MJ, Price GD. J Chem Phys 116 (2002) 7127.

Anderson OL. Physical Acoustics Vol. III-B, edited by Mason WP, New York: Academic Press, 1965.

Anderson OL, Dubrovinsky L, Saxena SK, LeBihan T. Geophys Res Lett 28 (2001) 399 .

Arroyave R, Shin D, Liu ZK. Acta Mater 53 (2005) 1809.

Aurelio G, Guillermet AF, Cuello GJ, Campo J. Metall Mater Trans A 33 (2002) 1307.

Aurelio G, Guillermet AF, Cuello GJ, Campo J. Metall Mater Trans A 34 (2003) 2771.

Baroni S, Giannozzi P, Testa A. Phys Rev Lett 58 (1987) 1861.

Baroni S, de Gironcoli S, Dal Corso A, Giannozzi P. Rev Mod Phys 73 (2001) 515. Bose SK, Dolgov OV, Kortus J, Jepsen O, Andersen OK. Phys Rev B 67 (2003) 214518.

Connolly JWD, Williams AR. Phys Rev B 27 (1983) 5169.

Debernardi A, Alouani M, Dreysse H. Phys Rev B 63 (2001) 064305.

de Wijs GA, Kresse G, Gillan MJ. Phys Rev B 57 (1998) 8223.

Dugdale JS, MacDonald DKC. Phys Rev 89 (1953) 832.

Ghosh G, Asta M. Acta Mater, in press, 2005.

Grad GB, Pieres JJ, Guillermet AF, Cuello GJ, Mayer RE, Granada JR. Z Metallkd 86 (1995) 395.

Grimvall G. Thermophysical Properties of Materials. Amsterdam: North-Holland, 1999.

Grover R, Getting IC, Kennedy GC. Phys Rev B 7 (1973) 567.

Guillermet AF. Intern J Thermophys 8 (1987) 481.

Guillermet AF. Trita-Mac-0325. Royal Institute of Technology, Sweden, 1987.

Herper HC, Hoffmann E, Entel P. Phys Rev B 60 (1999) 3839.

Hillert M, Jarl M. Calphad 2 (1978) 227.

Hohenberg P, Kohn W. Phys Rev 136 (1964) B864.

Inden G. Proc. Calphad V. Dusseldorf, 1976.

Jiang C, Chen LQ, Liu ZK. Acta Mater 2005, in press.

Jóhannesson GH, Bligaard T, Ruban AV, Skriver HL, Jacobsen KW, and Nørskov JK. Phys Rev Lett 88 (2002) 255506. Http://databases.fysik.dtu.dk/ 
Kohn W, Sham LJ. Phys Rev 140 (1965) A1133.

Korzhavyi PA, Ruban AV, Lozovoi AY, Vekilov YK, Abrikosov IA, Johansson B. Phys Rev B 61 (2000) 6003.

Mayer B, Anton H, Bott E, Methfessel M, Sticht J, Harris J, Schmidt PC. Intermetallics 11 (2003) 23.

Moruzzi VL, Janak JF, Schwarz K. Phys Rev B 37 (1988) 790.

Ostanin SA, Trubitsin VY. Phys Rev B 57 (1998) 13485.

Prinz GA. Phys Rev Lett 54 (1985) 1051.

Quong A, Liu AY. Phys Rev B 56 (1997) 7767.

Reeber RR. Phys Stat Solidi A 32 (1975) 321.

Sanchez JM, Stark JP, Moruzzi VL. Phys Rev B 44 (1991) 5411.

Skriver HL. Phys Rev B 31 (1985) 1909.

Slater JC. Introduction to Chemical Physics. New York: McGraw-Hill, 1939.

Soven P. Phys Rev 156 (1967) 809.

Taga A, Vitos L, Johansson B, Grimvall G. Phys Rev B 71 (2005) 014201.

van de Walle A, Ceder G. Rev Mod Phys 74 (2002) 11.

Vashchenko VY, Zubarev VN. Sov Phys Solid State 5 (1963) 653.

Vitos L, Abrikosov IA, Johansson B. Phys Rev Lett 87 (2001) 156401.

Wang K, Reeber RR. J Mater Res 11 (1996a) 1800.

Wang K, Reeber RR. High Temp Mater Sci 35 (1996b) 181.

Wang K, Reeber RR. Mater Sci Eng R23 (1998) 101.

Wang Y, Ahuja R, Johansson B. Intern J Quantum Chem 96 (2004a) 501.

Wang Y, Curtarolo S, Jiang C, Arroyave R, Wang T, Ceder G, Chen LQ, Liu ZK.

Calphad 28 (2004b) 79.

Wang Y, Liu ZK, Chen LQ. Acta Mater 52 (2004c) 2665.

Wasserman E, Stixrude L, Cohen RE. Phys Rev B 53 (1996) 8296.

Wolf W, Podloucky R. Phil Mag B 79 (1999) 839.

Xie JJ, de Gironcoli S, Baroni S, Scheffler M. Phys Rev B 59 (1999) 965.

Xie JJ, Chen SP, Brand HV, Rabie RL. J Phys Condens Matter 12 (2000) 8953.

Zharkov VN, Kalinin VA. Equations of State for Solids at High Pressures and Temperatures. New York: Consultants Bureau, 1971.

Zunger A, Wei SH, Ferreira LG, Bernard JE. Phys Rev Lett 65 (1990) 353. 


\section{Acknowledgements}

This work was carried out within the Centre of Computational Thermodynamics (CCT) financed by the Swedish Foundation for Strategic Research (SSF) and Swedish industry. A part of the work was supported by MATOP project in Brinell Centre, KTH.

This work bears the imprint of many people. Firstly I would like to thank my supervisor Professor Bo Sundman. He gives me excellent advices, guidance and freedom to express my scientific interests. He continuously encourages me to attend conferences and discuss with experts in different areas. I would also thank my co-supervisor Dr. Malin Selleby, who provides me the opportunity to work in the CCT project and always has patience reading my manuscripts word by word. I benefit greatly from scientific atmosphere in this group, and the collaborations within the CCT project organized by Dr. Malin Selleby.

I would appreciate the valuable discussions with Prof. John Ågren, Dr. Qing Chen, Dr. Pavel Korzhavyi, Prof. Emeritus Mats Hillert, Prof. Göran Grimvall, Prof. Börje Johansson and Dr. Karin Frisk. I am deeply impressed by their scientific enthusiasm and prudence during the research studies.

My great debts are also to my colleagues and friends in the department, especially David Andersson for help on using VASP, Henrik Strandlund and Klara Asp for mathematic assistances, Dr. Lars Höglund and Samuel Hallström for solving computer and network problems, Rosa Jerlerud, Huahai Mao and Dr. Lidong Teng for discussions on various problems, my earlier roommate Dr. Anders Pettersson and Dr. Joakim Odqvist for sharing knowledge and laugh.

Special thanks are given to my wife and my son. Without their supports and love, this work would not be possible to finish. Without their accompaniments, my life will be humdrum. Life does not always mean happiness, but I am glad that they are always there to share with me all the experiences. 